Aleksandra Katafiasz, Department of Mathematics, Pedagogical University, Chodkiewicza 30, 85-064 Bydgoszcz, Poland email: wspb04@@cc.uni.torun.pl Tomasz Natkaniec, Department of Mathematics, Gdańsk University, Wita Stwosza 57, 80-952 Gdańsk, Poland email: mattn@@ksinet.univ.gda.pl

\title{
A NEW VARIANT OF BLUMBERG'S THEOREM
}

\begin{abstract}
We prove that for every real function $f$ defined on a separable, complete and dense in itself metric space $X$ there exists a $c$-dense set $W \subset X$ such that $f \uparrow W$ is super quasi-continuous.
\end{abstract}

Our terminology is standard. We shall consider only real-valued functions defined on topological spaces. No distinction is made between a function and its graph. Symbol card $(X)$ will stand for the cardinality of a set $X$. The cardinality of $\mathbb{R}$ is denoted by $2^{\omega}$. For a cardinal number $\kappa$ we will write $\operatorname{cf}(\kappa)$ for the cofinality of $\kappa$. For a metric space $X, x \in X$ and $\varepsilon>0$ we denote by $B(x, \varepsilon)$ the open ball in $X$ centered at $x$ and with the radius $\varepsilon$. The set of all points at which a function $f: X \rightarrow \mathbb{R}$ is continuous (discontinuous) will be denoted by $C_{f}\left(D_{f}\right)$. The class of all continuous functions defined on $X$ will be denoted by $\mathrm{C}(X)$.

Recall also the following definitions ( $X$ is a topological space):

- $f: X \rightarrow \mathbb{R}$ is a pointwise discontinuous (shortly, $f \in \operatorname{PWD}(X)$ ) if the set $C_{f}$ in dense in $X$;

- $f: X \rightarrow \mathbb{R}$ is cliquish (shortly, $f \in \operatorname{CLIQ}(X)$ ) if for each $x_{0} \in X, \varepsilon>0$ and a neighborhood $W$ of $x_{0}$ there is a non-empty open set $W_{0} \subset W$ such that $\operatorname{osc} f\left\lceil W_{0}<\varepsilon\right.$;

- $f: X \rightarrow \mathbb{R}$ is quasi-continuous (shortly, $f \in \mathrm{QC}(X)$ ) if for each $x_{0} \in X$, $\varepsilon>0$ and a neighborhood $W$ of $x_{0}$ there is a non-empty open set $W_{0} \subset$ $W$ such that $\left|f\left(x_{0}\right)-f(x)\right|<\varepsilon$ for $x \in W_{0}$;

Key Words: continuous function, quasi-continuous function, super quasi-continuous function, cliquish function, pointwise discontinuous function, $\kappa$-Lusin set

Mathematical Reviews subject classification: Primary: 26A15 Secondary: 54C30

Received by the editors June 12, 1996 
- $f: X \rightarrow \mathbb{R}$ is super quasi-continuous (shortly, $f \in \mathrm{QC}^{*}(X)$ ) if $f\left\lceil C_{f}\right.$ is dense in $f$.

The relationships between those classes are well-known. (See, e.g., [6].) In particular, for a topological space the following inclusions hold:

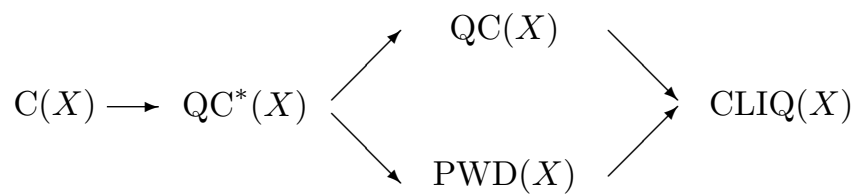

Generally, all those inclusions are proper. Nevertheless, for a complete metric space $X$ we have the following relations:

$$
\mathrm{C}(X) \rightarrow \mathrm{QC}^{*}(X)=\mathrm{QC}(X) \rightarrow \operatorname{CLIQ}(X)=\operatorname{PWD}(X) .
$$

In $1922 \mathrm{H}$. Blumberg proved the following theorem:

Theorem 1 [1] If $X$ is a complete metric space, then for every $f: X \rightarrow \mathbb{R}$ there exists a dense set $D \subset X$ such that $f\lceil D \in \mathrm{C}(D)$.

This theorem was extended in many directions and by many authors. (See [3] or [4] for the history of this study.) For example, it is known that the set $D$ in Blumberg's construction is countable, and, generally, one cannot increase the size of this set. (See [9].) In 1971 J. Brown proved the following strengthened form of Blumberg's theorem.

Theorem 2 [2] If $X$ is a complete metric dense in itself space, then for every $f: X \rightarrow \mathbb{R}$ there exists a c-dense set $X_{0} \subset X$ such that $f\left\lceil X_{0} \in \operatorname{PWD}\left(X_{0}\right)\right.$.

Brown's theorem yields to the following result.

Corollary 1 If $X$ is a complete metric dense in itself space, then for every $f: X \rightarrow \mathrm{I} R$ there exists a c-dense set $X_{0} \subset X$ such that $f\left\lceil X_{0}\right.$ is cliquish.

Following Brown, in this note we improve his result in the class of separable metric spaces by showing that for every $f: X \rightarrow \mathbb{R}$ there exists a c-dense set $X_{0} \subset X$ such that $f \uparrow X_{0} \in \mathrm{QC}^{*}\left(X_{0}\right)$.

Suppose that $M$ is a subset of a metric space $X$ and $\kappa$ is a cardinal number. We say that $M$ is a $\kappa$-Lusin set if $M$ has no nowhere dense subsets of cardinality $\kappa$. Usually, $\omega_{1}$-Lusin sets and $2^{\omega}$-Lusin sets are called Lusin sets and $c$-Lusin 
sets, respectively. It is well known that each Lusin set is of the second category. (See e.g., [7] or [5].) Every Lusin set is also $c$-Lusin. Moreover, if Continuum Hypothesis $(\mathrm{CH})$ holds, then every $c$-Lusin set is also a Lusin set. However, it is consistent that these notions are not equivalent. Indeed, e.g., under Martin's Axiom (MA) and the failure of $\mathrm{CH}$ there are $c$-Lusins sets on $\mathbb{R}$ which are not Lusin [5]. Then, for each cardinal $\kappa \leq 2^{\omega}$ with $\operatorname{cf}(\kappa)>\omega$ there are $\kappa$-Lusin sets in $\mathbb{R}$ which are not Lusin. (Indeed, under MA every set of reals with cardinality less than $2^{\omega}$ is meager [8], so it is enough to take a subset $L_{0}$ of $L$ with $\operatorname{card}\left(L_{0}\right)=\kappa$.)

Moreover, recall some topological notions, which were introduced in [2].

- $M$ is of a first $\kappa$-type iff $M$ is the union of a first category set and a $\kappa$-Lusin set;

- $M$ is of a second $\kappa$-type if $M$ is not of a first $\kappa$-type;

- if $G$ is an open subset of $X$, then the statement that $M$ is $\kappa$-typically dense in $G$ means that if $T$ is a non-empty open subset of $G$, then $T \cap M$ is of a second $\kappa$-type;

- $M$ is $\kappa$-typically dense at a point $x_{0} \in X$ iff $M \cap U$ is of a second $\kappa$-type for every neighborhood $U$ of $x_{0}$.

- $M$ is $\kappa$-typically dense in itself iff $M$ is $\kappa$-typically dense at every $x \in M .{ }^{1}$

Lemma 1 Assume that $\kappa$ is a cardinal number with uncountable cofinality. Then the family of all sets of a first $\kappa$-type forms a $\sigma$-ideal.

Lemma 2 Assume that $\kappa$ is a cardinal number such that $\omega<\operatorname{cf}(\kappa) \leq \kappa \leq 2^{\omega}$, $X$ is a separable metric space which is $\kappa$-typically dense in itself, $N \subset X$ is $\kappa$-typically dense in $X$ and $f: X \rightarrow \mathbb{I}$. Then there exists a $\kappa$-typically dense in $X$ set $N_{0} \subset N$ which satisfies the following condition:

(*) for every open set $W \subset \mathbb{I} R$ the set $N_{0} \cap f^{-1}(W)$ is $\kappa$-typically dense in itself.

Proof. Let $\left(B_{n}\right)_{n=1}^{\infty}$ and $\left(R_{n}\right)_{n=1}^{\infty}$ be countable bases of $X$ and $\mathbb{R}$, respectively. For each positive integers $n$ and $k$ put $D_{n, k}=N \cap B_{n} \cap f^{-1}\left(R_{k}\right)$. Let $D$ be the union of such $D_{n, k}$ that are of a first $\kappa$-type. By Lemma $1, D$ is also of a first $\kappa$-type. Set $N_{0}=N \backslash D$. Then $N_{0}$ is $\kappa$-typically dense in $X$ and it satisfies the condition $(*)$.

\footnotetext{
${ }^{1}$ Note that the empty set is always $\kappa$-typically dense in itself.
} 
Lemma 3 Assume that $a<b, \kappa$ is a cardinal number with $\omega<\operatorname{cf}(\kappa) \leq \kappa \leq$ $2^{\omega}, f$ is a real valued function a domain of which is a $\kappa$-typically dense subset $M$ of an open subset $G$ of a separable metric space $X$ and $f(x) \in(a, b)$ for each $x \in M$. Then there is a subset $N$ of $M$ such that $N$ satisfies the condition (*) (therefore $N$ is $\kappa$-typically dense in $G$ ) and $f\lceil N$ is continuous at some element of $N$.

Proof. In the same way as in the proof of Lemma 8 in [2] we can prove that there exists a subset $N$ of $M$ such that $N$ is $\kappa$-typically dense in $G$ and $f\lceil N$ is continuous at some $x_{0} \in N$. By Lemma 2 we may assume that $N$ satisfies the condition $(*)$.

Lemma 4 [2, Lemma 1] Assume that $\Phi$ is a property and every open subset of a metric space $X$ has an open subset with property $\Phi$. Then there exists a collection $\mathcal{G}$ of pairwise disjoint open subsets of $X$ such that $\bigcup \mathcal{G}$ is dense in $X$ and every set in $\mathcal{G}$ has property $\Phi$.

Theorem 3 Assume that $\kappa$ is a cardinal number such that $\omega<\operatorname{cf}(\kappa) \leq \kappa \leq$ $2^{\omega}$ and $X$ is a separable metric space which is $\kappa$-typically dense in itself. Then for every function $f: X \rightarrow \mathbb{R}$ there exists a $\kappa$-dense subset $W$ of $X$ such that $f\lceil W$ is super quasi-continuous. Therefore, $f\lceil W$ is quasi-continuous.

Proof. Let $\mathcal{R}$ be a countable base of $\mathbb{R}$. By Lemma 4 , there exists a family $\mathcal{G}_{1}$ of pairwise disjoint open subsets of $X$ such that $\bigcup \mathcal{G}_{1}$ is dense in $X$ and for each $G \in \mathcal{G}_{1}$ there is an $R_{G} \in \mathcal{R}$ such that $\operatorname{diam}\left(R_{G}\right)<1$ and $M_{G}=f^{-1}\left(R_{G}\right) \cap G$ is $\kappa$-typically dense in $G$. Now we define inductively an infinite sequence of steps such that each step involves four stages:

Step A1. Let $\mathcal{G}_{1}$ be the collection described above and for each $G \in \mathcal{G}_{1}$ let $R_{G}$ and $M_{G}$ be as described above.

Step B1. For each $G \in \mathcal{G}_{1}$, let $N_{G}$ be a subset of $M_{G}$ described in Lemma 3, which satisfies the condition (*) from Lemma 2 and let $x_{G} \in N_{G} \cap C_{f\left\lceil N_{G}\right.}$.

Step C1. For $G \in \mathcal{G}_{1}$, let $H_{G}$ be a nowhere dense subset of $N_{G}$ such that $x_{G} \in H_{G}$, card $\left(H_{G}\right) \geq \kappa$ and let $\mathcal{K}_{G}$ be a collection of open balls such that

(i) $\operatorname{diam}(B)<1$ for each $B \in \mathcal{K}_{G}$;

(ii) sets in $\mathcal{K}_{G}$ are pointwise disjoint;

(iii) $\bigcup \mathcal{K}_{G} \subset G \backslash H_{G}$ and $\bigcup \mathcal{K}_{G}$ is dense in $G$; 
(iv) for each $B \in \mathcal{K}_{G}$ there exists $R_{B} \in \mathcal{R}$ such that $R_{B} \subset R_{G}$, $\operatorname{diam}\left(R_{B}\right)<\frac{1}{2}$ and the set $B \cap N_{G} \cap f^{-1}\left(R_{B}\right)$ is $\kappa$-typically dense in $B$;

(v) for every $x \in H_{G}$ and for each open neighborhood $W \subset X \times \mathbb{R}$ of $(x, f(x))$ there exists a $B \in \mathcal{K}_{G}$ such that $B \times R_{B} \subset W$.

The construction of $\mathcal{K}_{G}$. Let $\mathcal{U}$ be a countable base of $X$ and let $\left(U_{n} \times R_{n}\right)_{n}$ be a sequence of all products $U \times R$ where $U \in \mathcal{U}, R \in \mathcal{R}$ and $\left(f\left\lceil H_{G}\right) \cap(U \times R) \neq \emptyset\right.$. Inductively choose a ball $B_{n}$ such that $\operatorname{cl}\left(B_{n}\right) \subset U_{n} \backslash\left(\operatorname{cl}\left(H_{G}\right) \cup \bigcup_{m<n} \operatorname{cl}\left(B_{m}\right)\right)$ and $f^{-1}\left(R_{n}\right) \cap B_{n} \cap N_{G}$ is $\kappa$ typically dense in $B_{n}$. (It is possible because $N_{G} \cap f^{-1}\left(R_{n}\right)$ is non-empty and, by $(*), \kappa$-typically dense in itself, and $U_{n} \backslash\left(\operatorname{cl}\left(H_{G}\right) \cup \bigcup_{m<n} \operatorname{cl}\left(B_{m}\right)\right)$ is an open neighborhood of some $x \in N_{G} \cap f^{-1}\left(R_{n}\right)$.)

Let $\mathcal{K}^{\prime}{ }_{G}=\left\{B_{n}: n \in \mathbb{N}\right\}$ and $R_{B}=R_{n}$ for $B=B_{n}$. Then the conditions (i)-(iv) are evident except the statement $\cup \mathcal{K}^{\prime}{ }_{G}$ is dense in $G$. By Lemma 4 this family can be extended to a family $\mathcal{K}_{G}$ what satisfies statements (i)-(iv). Now we shall verify (v). Fix $x \in H_{G}$ and an open set $W \subset X \times \mathbb{R}$ such that $(x, f(x)) \in W$. Then there exists $n \in \mathbb{N}$ such that $(x, f(x)) \in U_{n} \times R_{n} \subset W$. Thus $B_{n} \times R_{n} \subset W$.

Step D1. For $G \in \mathcal{G}_{1}$ and for each $B \in \mathcal{K}_{G}$, put $M_{B}=N_{G} \cap B \cap f^{-1}\left(R_{B}\right)$.

Now, for each $n>1$, steps An, Bn, Cn and Dn are defined as follows:

Step An. Let $\mathcal{G}_{n}=\bigcup\left\{\mathcal{K}_{G}: G \in \mathcal{G}_{n-1}\right\}$.

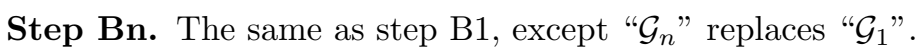

Step Cn. The same as step $\mathrm{C} 1$, except " $\mathcal{G}_{n}$ " replaces " $\mathcal{G}_{1}$ " and " $\frac{1}{n}$ " replaces "1".

Step Dn. The same as step D1, except " $\mathcal{G}_{n}$ " replaces " $\mathcal{G}_{1}$ ".

Now, set $W=\bigcup_{n=1}^{\infty} \bigcup_{G \in \mathcal{G}_{n}} H_{G}$ and $C=\left\{x_{G}: G \in \bigcup_{n=1}^{\infty} \mathcal{G}_{n}\right\}$. As in [2] we can observe that $W$ is $\kappa$-dense in $X$ and $C$ is dense in $X$. Indeed, for $x_{0} \in X$ and $\varepsilon>0$ let $n$ be a positive integer such that $\frac{1}{n}<\frac{\varepsilon}{3}$. Since $\bigcup \mathcal{G}_{n}$ is dense in $X$, there exists $G \in \mathcal{G}_{n}$ such that $G \cap B\left(x_{0}, \frac{1}{n}\right)^{n} \neq \emptyset$. Since $\operatorname{diam}(G)<\frac{1}{n}$, $G \subset B\left(x_{0}, \varepsilon\right)$ and $H_{G}$ is a subset of $W \cap B\left(x_{0}, \varepsilon\right)$ with $\operatorname{card}\left(H_{G}\right) \geq \kappa$. Moreover, $x_{G} \in C \cap B\left(x_{0}, \varepsilon\right)$.

Now, suppose that $x \in C$. There exist $n \in \mathbb{N}$ and $G \in \mathcal{G}_{n}$ such that $x=x_{G}$. Then $W \cap G \subset N_{G} \cap G$ and $f\left\lceil N_{G}\right.$ is continuous at $x_{G}$, so $f \nmid W$ is continuous at $x$. 
To verify that $f\left\lceil C\right.$ is dense in $f \uparrow W$, fix $x_{0} \in W$ and $\varepsilon>0$. There exists $n \in \mathbb{N}$ and $G \in \mathcal{G}_{n}$ such that $x_{0} \in H_{G}$. By the statement (v) of Step Cn, there is $B \in \mathcal{K}_{G}$ such that $B \subset B\left(x_{0}, \varepsilon\right)$ and $f\left(N_{B}\right) \subset\left(f\left(x_{0}\right)-\varepsilon, f\left(x_{0}\right)+\varepsilon\right)$. Then $x_{B} \in C \cap B\left(x_{0}, \varepsilon\right)$ and $f\left(x_{B}\right) \in\left(f\left(x_{0}\right)-\varepsilon, f\left(x_{0}\right)+\varepsilon\right)$, which completes the proof.

Because any complete metric space which is dense in itself is $2^{\omega}$-typically dense in itself [2, Corollary] and $\operatorname{cf}\left(2^{\omega}\right)>\omega$, we have the following:

Corollary 2 If $X$ is a separable, complete, dense in itself metric space, then for every function $f: X \rightarrow I R$ there exists a c-dense set $W \subset X$ such that $f \uparrow W$ is super quasi-continuous.

Now we shall consider metric spaces for which Corollary 2 does not hold, even in a weaker form.

Lemma 5 Assume that $\operatorname{cf}(\kappa)>\omega, L \subset X$ is a $\kappa$-Lusin set and $f: L \rightarrow I R$ is cliquish. Then the set $D_{f}$ has cardinality less than $\kappa$.

Proof. Recall that $D_{f}=\bigcup_{n=1}^{\infty} D_{f, n}$, where $D_{f, n}=\left\{x \in L: \operatorname{osc} f(x) \geq \frac{1}{n}\right\}$. Because every set $D_{f, n}$ is closed in $L$, so either $D_{f, n}$ is nowhere dense and $\operatorname{card}\left(D_{f, n}\right)<\kappa$ or $\operatorname{int}_{L}\left(D_{f, n}\right) \neq \emptyset$. The second case is impossible, because $f$ is cliquish. Thus card $\left(D_{f, n}\right)<\kappa$ for each $n$, so card $\left(D_{f}\right)<\kappa$.

Theorem 4 Let $X$ be a separable metric space. If $X$ is not $2^{\omega}$-typically dense in itself, then there exists a function $f: X \rightarrow I R$ such that $f\lceil W$ is cliquish for no $2^{\omega}$-dense in $X$ set $W$.

Proof. We can assume that every open subset of $X$ has cardinality at least $2^{\omega}$.

Let $G$ be a non-empty open subset of $X$ such that $G$ is of first $2^{\omega}$-type. Then there are: a $2^{\omega}$-Lusin set $L$ and a family of pairwise disjoint nowhere dense sets $\left\{M_{i}\right\}_{i}$ such that $L \cap \bigcup_{i} M_{i}=\emptyset$ and $G=L \cup \bigcup_{i} M_{i}$. As in the proof of Theorem 2 in [2] we consider two cases: if $G$ is of the first category (or $L$ has cardinality less than $2^{\omega}$ ), and if there exists an open subset $T$ of $G$ such that $L$ is dense in $T$. In both those cases we define $f$ in the same way as in [2]. In the last part of the proof of the second case we use Lemma 5 to observe that the supposition that $f$ is cliquish implies the continuity of $f$ on a set of size $2^{\omega}$, which is impossible.

Corollary 3 Assume that $X$ is a separable dense in itself metric space. Then for $\kappa=2^{\omega}$ the following conditions are equivalent: 
(i) for each function $f: X \rightarrow \mathbb{R}$ there exists a $\kappa$-dense set $W \subset X$ such that $f \nmid W \in \mathrm{QC}^{*}(W)$;

(ii) for each function $f: X \rightarrow \mathbb{R}$ there exists a $\kappa$-dense set $W \subset X$ such that $f \nmid W \in \operatorname{PWD}(W)$;

(iii) for each function $f: X \rightarrow I R$ there exists a $\kappa$-dense set $W \subset X$ such that $f \uparrow W \in \mathrm{QC}(W)$

(iv) for each function $f: X \rightarrow I R$ there exists a $\kappa$-dense set $W \subset X$ such that $f \nmid W \in \operatorname{CLIQ}(W) ;$

(v) $X$ is $\kappa$-typically dense in itself.

\section{Questions.}

1. Does there exist a metric space $X$ and a cardinal $\kappa$ for which the conditions (i) - (iv) are not equivalent?

2. Assume that $X$ is a separable dense in itself metric space. Are the conditions (i) - (v) equivalent for $\kappa \in\left(\omega, 2^{\omega}\right)$ ?

Obviously, if $\mathrm{CH}$ is true then the notions of typically dense and $c$-typically dense are the same. So one can suppose that if $X$ is $\kappa$-typically dense in itself for some uncountable cardinal $\kappa$ then $X$ is $c$-typically dense in itself. The next proposition shows that this hypothesis is not true.

Proposition 1 Assume that $M A$ is true and $C H$ fails. Then there exists a subspace $X \subset \mathbb{I} R$ that is $\kappa$-typically dense in itself for each $\kappa<2^{\omega}$ with $\operatorname{cf}(\kappa)>\omega$, but not $2^{\omega}$-typically dense.

Proof. Let $X$ be a $c$-Lusin set that is $c$-dense in $\mathbb{R}$. (See, e.g., [5].) Then $X$ is not $2^{\omega}$-typically dense. We shall verify that it is $\kappa$-typically dense for a fixed $\kappa<2^{\omega}$ with uncountable cofinality. Suppose that there exists a non-empty open in $X$ set $G$ that is a first $\kappa$-type set in $X$. So, in $X$ there are a $\kappa$-Lusin set $L$ and a meager set $A$ such that $G=L \cup A$. Then $L$ is a $c$-Lusin set and $A$ is meager in $\mathbb{R}$. Thus card $(A)<2^{\omega}$ and consequently, card $(L)=2^{\omega}$. Since $X$ is $c$-dense in itself, every meager in $\mathbb{R}$ subset $B$ of $L$ is also meager in $X$. Therefore every set $B \subset L$ with card $(B)=\kappa$ is meager in $X$ (cf., [8]), contrary to the definition of $\kappa$-Lusin set.

Corollary 4 Assuming MA $+\neg \mathrm{CH}$ and $2^{\omega}$ is not the successor of $\kappa$ with $\operatorname{cf}(\kappa)=\omega$, there exists a subspace $X \subset \mathrm{I} R$ such that: 
1. for each $\kappa<2^{\omega}$ and $f: X \rightarrow I R$ there exists a $\kappa$-dense in $X$ set $W_{\kappa}$ such that $f\left\lceil W_{\kappa} \in \mathrm{QC}^{*}\left(W_{\kappa}\right)\right.$;

2. there is $f: X \rightarrow \mathbb{R}$ such that $f\lceil W \in \operatorname{CLIQ}(W)$ for no $c$-dense in $X$ set $W$.

\section{References}

[1] H. Blumberg, New properties of all real functions, Trans. Amer. Math. Soc., 24 (1922), 113-128.

[2] J. B. Brown, Metric spaces in which a strengthened form of Blumberg's theorem holds, Fund. Math., 71 (1971), 244-253.

[3] J. B. Brown, Variations on Blumberg's theorem Real Anal. Exchange 9 (1983-84), 123-137.

[4] J. B. Brown, Restriction Theorems in Real Analysis, Real Anal. Exchange 20 (1994-95), 510-526.

[5] A. W. Miller, Special subsets of the real line, in Handbook of Set-Theoretic Topology, edited by K. Kunen and J. E. Vaughan, North-Holland (1984), 201-233.

[6] T. Neubrunn, Quasi-continuity, Real Anal. Exchange 14 (1988-89), 259307.

[7] J.C. Oxtoby, Measure and Category, New York-Heidelberg-Berlin, 1971.

[8] J. Shoenfield, Martin's axiom, Amer. Math. Monthly 82 (1975), 610-617.

[9] W. Sierpiński and A. Zygmund, Sur une fonction qui est discontinue sur tout ensemble de puissance du continu, Fund. Math., 4 (1923), 316-318. 